\title{
Alcohol use disorders in people with intellectual disability
}

Fionnuala Williams, Christos Kouimtsidis \& Alexander Baldacchino

Fionnuala Williams is an ST5 in psychiatry of intellectual disability, working at Lynebank Hospital in south-east Scotland. She has spent her special interest sessions in the Fife addiction services. Christos Kouimtsidis is a consultant psychiatrist in addictions with Surrey and Borders Partnership NHS Foundation Trust, and an honorary clinical senior lecturer at Imperial College London. His research interests include psychological interventions and hybrid models in addiction, the effect of alcohol detoxifications on the brain and alcohol clinical interventions for special populations. Alexander Baldacchino is a professor of psychiatry and addictions at St. Andrews University Medical School. $\mathrm{He}$ is also a clinical lead and consultant in addiction psychiatry in NHS Fife.

Correspondence Dr Fionnuala Williams, Lynebank Hospital, Halbeath Road, Dunfermline, Fife KY11 4UW, UK. Email: fionnuala. williams@nhs.net

\section{Copyright and usage}

(C) The Royal College of Psychiatrists 2018

\section{SUMMARY}

This article initially highlights that although the prevalence of alcohol use disorders in people with intellectual disability (PWID) appears to be low, it is a significant issue. This group can be more vulnerable to the adverse effects of alcohol and it is likely that many PWID who have alcohol use disorders are not being identified. We go on to review the limited existing literature on treatment for PWID who have alcohol use disorders and the challenges in meeting the needs of this patient group. We explore how assessment and treatment of alcohol use disorders in this population can be and needs to be tailored to the needs of PWID on an individual basis. There is also discussion about the use of incapacity legislation to treat this group.

\section{LEARNING OBJECTIVES}

- Be aware that alcohol use disorders can be especially problematic for PWID, that such disorders can often go undetected and that adapted screening techniques may be needed to identify such problems

- Understand the difficulties that this population has in accessing addiction services and that successful management of PWID who misuse alcohol is usually dependent on appropriate joint working between intellectual disability and addiction services

- Be aware that PWID are not a homogeneous group, rather they vary widely in their abilities, necessitating interventions tailored to the individual, and that the use of compulsory measures to manage PWID who lack capacity regarding to their alcohol use should be done with caution

\section{DECLARATION OF INTEREST}

None.

With the move toward community care in the late 20th century, people with intellectual disability (PWID) now have more freedom over how they conduct their lives (Degenhardt 2000). This also includes greater access to alcohol and illicit drugs (Huxley 2005). Alcohol is the most misused intoxicating substance in this population group (McGillivray 2001; Taggart 2006; Chaplin 2011;
VanDerNagel 2011; To 2014; Bhandari 2015). This article aims to look at alcohol use disorders (both harmful and dependent use) in PWID. It does not encompass other neurodevelopmental disorders commonly comorbid with intellectual disability, such as Autism Spectrum Disorder. Studies have indicated that PWID develop alcohol use disorders for reasons similar to the general population, with primary reasons being psychological trauma (e.g. multiple bereavements, rape and physical, emotional and financial abuse) and social distance from the community (e.g. isolation and lack of friendship and companionship) (Taggart 2006). These alcohol use disorders are subsequently associated with increased problems with the physical, psychological and social well-being of PWID (Huxley 2005).

\section{Prevalence of alcohol use disorders in PWID}

The true extent of the problem is unclear as there are only limited data regarding the prevalence and severity of alcohol use disorders in PWID (Huxley 2005), and the available data is conflicting: one early study indicated that PWID may be overrepresented in addiction services (Westermeyer 1996), whereas others have found no significant difference in rates of alcohol use disorders compared with those without intellectual disability (VanDerNagel 2011; Bhandari 2015, McGillivray 2016). However, a number of studies (Robertson 2000; Asscher 2012), including a large, comprehensive population-based UK study (Cooper 2007), suggest that prevalence is low compared to the general population. This study found a prevalence of alcohol/substance use disorder of $1 \%$ on clinical diagnosis. In the mild intellectual disability group, $1.8 \%$ had an alcohol/substance use disorder. In the moderate-to-profound intellectual disability group, $0.5 \%$ had an alcohol/substance use disorder. The study itself does not make a comparison with a group without intellectual disability drawn from the same population, nor does it separate alcohol from substance use disorder in its results, making it difficult to determine how the prevalence of alcohol use disorders in PWID compares to the general population. However, the Scottish Health Survey in 2016 found that $26 \%$ of adults in 
BOX 1 Conditions with high rates of prevalence in PWID that are also linked to alcohol use disorders

- Epilepsy

- Gastro-oesophageal reflux disease

- Cancer

- Dementia

- Accidents

- Nutritional problems

(NHS Health Scotland 2004)

Scotland, were drinking more than the recommended maximum of 14 units per week. Although this is not directly comparable with the figures obtained by Cooper et al who looked at clinical diagnosis of both alcohol and substance use in only one area of Scotland, they suggest that the prevalence in PWID is significantly lower (McLean 2017).

However, findings from a more recent study conducted in a different region of the UK has found a much higher prevalence than the figures reported by Cooper et al in 2007. It screened a random sample of 40 patients from a psychiatric intellectual disability community service, using the CAGE (Cutting down, Annoyance by criticism, Guilty feeling, and Eye-openers) questionnaire (Ewing 1984) and the Alcohol Use Disorders Identification Test (AUDIT (Babor 2001)) to assess alcohol use (Pezzoni 2015). It reported $20 \%$ of patients as having an alcohol use disorder according to the CAGE (Cutting down, Annoyance by criticism, Guilty feeling, and Eye-openers) questionnaire (score $>2$ ), and $22.5 \%$ according to the AUDIT (score $>8$ ): figures which are much closer to those found from the general population in the Scottish Health Survey 2016. None of the patients had such a diagnosis recorded in their clinical notes (Pezzoni 2015). It should be noted however that this study also has limitations as these screening tools have not been formally validated for use with PWID, and as they are based on self-report, individuals may be over- or under-reporting their use of alcohol.

\section{Significance of the problem}

Even if the prevalence is low, the consequences of alcohol use disorders in PWID are significant. Continued misuse is likely to negatively affect successful community living (Huxley 2005; Barrett 2006). PWID are potentially more vulnerable to some of the physical consequences of alcohol use disorders. A number of physical health conditions (see
Box 1) that can also be linked to or exacerbated by alcohol use disorders already have higher rates in PWID, and can therefore be exacerbated further if PWID have an alcohol use disorder. To complicate matters further, in PWID these conditions are often not recognised or treated (Cooper 2004). Psychological and social consequences of alcohol use disorders for PWID are listed in Box 2 and a case vignette is described in Box 3. A study of PWID who did and did not present to the emergency department in crisis found that those who had alcohol or drug misuse problems were more likely to attend the emergency department in response to crisis than those experiencing any other life event (Lunsky 2011). A number of studies support a link between alcohol use disorders and offending in PWID (McGillivray 2001; Lindsay 2013), and indeed one study into PWID in custody found that $62 \%$ of criminal behaviour was related to excessive alcohol consumption (assessed for using AUDIT) (Scott 2006).

Evidence for whether PWID have sufficient knowledge, skills and personal resources regarding alcohol use disorders and the risks entailed is conflicting. One small study indicated that PWID were very knowledgeable about why alcohol was bad for their health and were able to make a choice to moderate unhealthy behaviour (Caton 2012). However, there are a number of larger studies in this field that either indicate a lack of knowledge in this area or difficulty in acting on this knowledge to make healthier choices about alcohol use (McCusker 1993; McGillivray 2001; Kuijken 2016); therefore, current evidence seems to primarily indicate that PWID are potentially at greater risk of use disorders than the general population. Indeed, some studies indicate that when PWID use substances, they are more inclined to misuse (McGillicuddy 1999; To 2014). In one study of 397 homeless people, participants with suspected intellectual disability (based on a screening test) had up to two times greater odds of being

BOX 2 Consequences of alcohol misuse relevant to PWID

$\begin{array}{ll}\text { Psychological } & \text { Social } \\ \text { Exacerbation of } & \text { - Exclusion from services as a result of behavioural difficulties } \\ \text { cognitive deficits } & \text { - Further marginalisation and exclusion } \\ \text { Mental health } & \text { - Greater barriers to accessing services } \\ \text { problems } & \text { - Increased risk of unemployment } \\ & \text { - Increased risk of poverty } \\ & \text { - Increased risk of offending }\end{array}$


Fred is a 24-year-old man with mild intellectual disability and dependent use of alcohol. Fred previously had his own tenancy but concerns were raised with social work about his ability to maintain this. He would invite people to his flat to drink with him who would then end up exploiting him for money or stealing his things. Fred found it difficult to safeguard himself from these people as he saw them as his friends. Fred was and continues to be a frequent attender at the emergency department with self- harm. This only ever occurs when he is under the influence of alcohol and once Fred sobers up, he is remorseful of his actions. He also occasionally gets into fights when he is under the influence, which has led to him experiencing a number of injuries. Fred has epilepsy and his alcohol use is leading to an increase in his seizure frequency. His family have been very concerned about his risky behaviour. He currently lives at home with his father who is struggling to continue to support him due to his ongoing drinking. His living situation is at the point of breaking down. Fred says he wants to continue drinking, but he has some insight into the effects of his drinking and that it would be helpful to stop. He is unsure of how to achieve this and has limited understanding of safe drinking limits. Attempts have been made to engage him with local mainstream alcohol services but his father states that the staff do not know how to work with people with an intellectual disability. classified as substance dependent than participants without intellectual disability (Van Straaten 2014). It has also been suggested that PWID are vulnerable to the effects of alcohol at a lower level than the general population (Westermeyer 1996), and that screening tools should be adapted and cut-off points modified (Pezzoni 2015). However, there has been no research into or even arbitrary suggestion as to what these lower levels should be.

In summary, the available evidence would suggest that alcohol use disorders are a problem in this population, albeit at a lower prevalence than in the general population. This is compounded by the potential lack of adequate skills to make healthy choices regarding dysfunctional behaviours such as substance misuse and their adverse consequences.

\section{PWID are not a homogeneous group}

Studies regarding alcohol use disorders in PWID have been affected by a number of limitations. A frequent limitation has been not considering the differences between levels of intellectual disability. PWID should not be considered a homogeneous group. As demonstrated by Cooper (2007), substance use disorders are more common in those with milder levels of intellectual disability and this is likely to be the case as they are more able to access the community. Descriptions of the functional ability expected in different levels of intellectual disability are given in Box 4. Patterns of substance use in PWID have also been found to vary because of other factors, as listed in Box 5. The situation is complicated further as there is variation in the way that people with mild intellectual disability are managed in different areas, as in some places they fall under the remit of general adult psychiatry.

Studies have also often looked at substance use disorders as a homogeneous concept rather than separating out different types of substance being used and different types of use disorder. Also many studies have used instruments designed for use with the general population which do not appear to have been validated for use with PWID (Barrett 2006; Asscher 2012; Bhandari 2015; McGillivray 2016). Although Kouimtsidis 2017 used a more accessible assessment instrument, it has not been formally validated for use with PWID (Kouimtsidis 2017). In both intellectual disability and alcohol use disorders, there is a spectrum of how the individual can be affected by each disorder, and thus when a patient presents with both conditions simultaneously, it is even more important to tailor intervention to the individual's need and abilities. As detailed below, both assessment and treatment of alcohol use disorders needs to be made accessible to PWID.

\section{Problems with accessibility}

Intellectual disability and alcohol use disorders are conditions that individually are associated with poor engagement in care and can go undetected. It is the authors' experience that PWID struggle to access support from addiction services and complete treatment programmes, and this is supported by the literature (Slayter 2010; McGillivray 2016). Interviews with ten PWID found that their main source of support was from community-level intellectual disability services but that although these services are helpful, they could do little to persuade them to change their hazardous drinking patterns (Taggart 2007). Most who had used mainstream addiction services had not found their input helpful, particularly disliked group work and felt that they had not been adequately supported with the negative life experiences underlying their substance misuse (Taggart 2007). Whilst the generalisability of this study to PWID as a whole is limited due the small sample, it corroborates our clinical experience that PWID usually do not find mainstream addiction services accessible. 
BOX 4 ICD-11 beta: Descriptions of functioning of people with different levels of intellectual disability

$\begin{aligned} & \text { Level of intellectual disability } \\ & \text { Mild }\end{aligned}$
$\begin{aligned} & \text { Level of functioning } \\ & \text { practical activities, and are able to live relatively independently. Capable of finding employment as adults, but they may need } \\ & \text { appropriate support. } \\ & \text { Moderate } \\ & \text { Capacity for academic skills and language comprehension varies; usually limited to basic skills. Some can manage basic self-care } \\ & \text { and domestic and practical activities. Most need significant ongoing support to live independently and maintain employment } \\ & \text { as adults. } \\ & \text { Very limited capacity for language and ability to gain academic skills, which is often accompanied by motor impairments. Daily } \\ & \text { support in a supervised environment is usually required, although some may develop basic self-care skills with intensive } \\ & \text { support and training. } \\ & \text { Very limited communication ability. Potential to gain academic skills is limited to basic concrete skills. Motor and sensory } \\ & \text { impairments are usually present, and daily support in a supervised care environment is usually required. }\end{aligned}$
Profound

(World Health Organization 2017)

There has been concern expressed regarding the ability of both addiction and intellectual disability services to meet the needs of PWID who have substance use disorders (Barrett 2006). Staff from intellectual disability services feel they lack training and expertise regarding the assessment, treatment and management of substance use disorders in PWID and in motivational interviewing (McLaughlin 2007; VanDerNagel 2011). The literature indicates that addiction services have limited experience and training in managing PWID (McLaughlin 2007) and staff have recognised that their services are not effectively set up to meet the needs of PWID (McLaughlin 2007). Staff in addiction services may assume that cognitive difficulties are related to the alcohol use disorder rather than the underlying intellectual disability, or they may not recognise that PWID can have significant difficulties in their ability to take in and retain information and adhering to treatment regimens (Huxley 2005). Addiction-trained staff have also been found to use the same assessment schedules and therapeutic interventions with PWID as they use in the general population (McLaughlin 2007), with only minor modifications to language and duration of the

BOX 5 Factors associated with substance use in PWID

- Psychiatric comorbidity, e.g. schizophrenia spectrum disorders (Taggart 2006; Chaplin 2011; VanDerNagel 2011; To 2014)

- Lack of daytime activities (Taggart 2006; VanDerNagel 2011; To 2014)

- Male gender (Taggart 2006; Chaplin 2011; To 2014)

- Forensic history (Chaplin 2011)

- Living independently (Taggart 2006; To 2014) intervention. PWID may therefore not be provided with the appropriate treatment strategy, which may need to be very different from that provided to most people that access addiction services (Huxley 2005). It has been recognised that addiction services in the UK are overstretched in providing services to the mainstream population, and that they therefore may struggle even more to meet the complex needs of PWID (Scott 2006; McLaughlin 2007).

There is also a difference in approach between intellectual disability and addiction services. There is some suggestion that intellectual disability services may take a more paternalistic role than addiction services, and that they need to provide people with enough information to make an informed choice about use and allow patients the opportunity for positive risk-taking (Huxley 2005). There is little evidence to suggest whether intellectual disability or addiction services are best placed to support this population (Huxley 2005). Increased liaison and joint working between the two services has been suggested (McLaughlin 2007), but there is evidence to indicate that this does not often work well in practice (VanDerNagel 2012; To 2014). It tends to be ad hoc, with professionals from intellectual disability services contacting their peers in addiction services for advice (McLaughlin 2007). Suggestions for improvement include the development of a link person/joint person between both services who could help devise a joint working strategy to ensure that the patient gets the right services. It was also suggested that intellectual disability staff could take on an educational role toward support services and carers to promote recognition and early intervention for people with intellectual disability and substance misuse problems (McLaughlin 2007).

Many people with alcohol use disorders are managed primarily by third-sector organisations. 
UK studies exploring how addiction services staff manage PWID have only included National Health Service addiction services, so it is unclear if third-sector organisations can meet the needs of this group. PWID with alcohol use disorders may also present to their general practitioner (GP). It has been identified previously that GPs have been shown to lack the skills and knowledge to identify, and offer effective care to people with substance issues (McGillion 2000), and that GPs lack experience in working with PWID (Scottish Government 2013). It would appear likely that primary care would struggle further in managing PWID who also have alcohol use disorders.

In terms of equality of care, addiction services should be as accessible to PWID as they are to the general population. However, given the importance of communication skills to be able to establish a successful therapeutic relationship, at present intellectual disability services are best placed to provide support to PWID in the first instance. It has been suggested that intellectual disability services upskill to take on the primary responsibility for PWID with alcohol use disorders to minimise potentially damaging disruption from patients being passed between services, and to allow for a broader view of an individual patient's situation (Huxley 2005). Given the reportedly low prevalence of alcohol use disorders in PWID, it may be difficult for staff in intellectual disability services to maintain these skills, although the same would also be true for addiction services training to support PWID. The authors would suggest that both sectors receive training in each other's discipline, but that intellectual disability services should take the primary supportive role. If there is dependent use (see Box 6), management of this would normally involve pharmacological treatment which should be administered by specialist staff (NICE 2011) thus involvement of the addiction services is essential. It may also be appropriate for intellectual disability

\section{BOX 6 ICD-10: Definition of alcohol dependency}

Three of the following:

- a strong desire to drink

- difficulties in controlling drinking

- persistent drinking despite harmful effects

- alcohol prioritised over other activities and obligations

- increasing tolerance

- physical symptoms of withdrawal

(World Health Organization 2010) services to liaise early with appropriate National Health Service and third-sector addiction services for support, even if there is no dependency in complex cases, or if the patient expresses a preference for this. The authors would suggest that community intellectual disability staff attend addiction services appointments with the patient and go over what has taken place in the appointments at a later date if necessary, to maximise understanding and engagement.

\section{Interventions}

There have been a number of articles written suggesting how interventions aimed at PWID should be conducted, as well as several studies evaluating some techniques put into practice, which will we summarise here. It is assumed by some that interventions aimed at the general population will also be suitable for PWID; however, because of the cognitive deficits PWID have, this is unlikely to be the case (Kouimtsidis 2015), and anecdotal data suggests that treatment will require modifications to meet the needs of PWID (Burgard 2000). Highly cognitively based therapies may not be suited to this patient group (Degenhardt 2000).

A number of adaptations to treatment programmes have been suggested in the literature. Sessions might be more frequent (Barrett 2006; Kouimtsidis 2017), longer (Kouimtsidis 2017) and well-structured (Degenhardt 2000) to allow them to be tailored to the difficulties faced by PWID, specifically regarding the comprehension of, assimilation of, and adaptation to new ideas. Providing individual sessions on top of group sessions (Forbat 1999; Barrett 2006), connecting new ideas with things already familiar to the patient and only progressing once skills in earlier stages are acquired (Degenhardt 2000) can also help in this regard. Use of easy-to-read materials (Barrett 2006) and video vignettes (McMurran 1993; Forbat 1999) may make treatment more accessible to PWID. Use of role playing (McGillicuddy 1999; Degenhardt 2000), practising by applying skills to examples of real-life situations (e.g. trip to the pub and refusing alcohol) (Forbat 1999; Degenhardt 2000), the therapist modelling desirable behaviours, use of rehearsal and repetition of new ideas (Degenhardt 2000; Barrett 2006), and demonstration and discussion of inappropriate behaviours (Degenhardt 2000) can help to consolidate learning by putting ideas into practice in a more tangible, and therefore memorable form. Learning can be enhanced by positive reinforcement such as rewards and praise (Degenhardt 2000), and by promoting support from carers/family (Barrett 2006). PWID often do not live completely independently of other people 
and unlike the general population, are more likely to have carers who could play an important role in helping them engage with treatment. Indeed, it has been shown that lack of carer support can adversely affect engagement with interventions (Kouimtsidis 2017).

It is also important to tackle other issues that may be contributing to the alcohol use disorder, such as teaching assertiveness skills (to reduce the effect of influence/exploitation by others) (McGillicuddy 1999), teaching coping skills and providing support to manage the effects of negative life experiences (to provide an alternative to coping by drinking) (Degenhardt 2000; Taggart 2007), and social support to help with isolation (to reduce the use of alcohol to seek social contact or cope with feelings of loneliness) (Taggart 2007). In a previous qualitative study, most PWID stated they would prefer oneto-one sessions rather than group sessions for discussing substance misuse and life circumstances (Taggart 2007), and this could also be more helpful to promote communication.

It is of utmost importance that PWID who have alcohol use disorders are identified by services to ensure they can receive treatment. The first author has conducted an evaluation of nurse-led health screening in her service and has found that the current screening methods are potentially missing people with intellectual disability who consume alcohol above recommended limits and could benefit from support. An easy-read form of the AUDIT has been developed for use with PWID, which, although it has not yet been formally validated for use in this population group, has been shown to be acceptable to this population (Kouimtsidis 2017).

There is limited evidence for interventions specifically targeted at PWID who have alcohol use disorders. Most studies have been uncontrolled, small, have taken place in secure settings rather than community settings and have used measures that are either not appropriate for PWID or have been modified but not validated (Kouimtsidis 2015). Very few have looked at how interventions have affected actual alcohol consumption, yet most have shown positive outcomes in terms of improvement in knowledge and skills (McCusker 1993; McMurran 1993; Forbat 1999; McGillicuddy 1999). Of note, one small study (Mendel 2002) has demonstrated that motivational interviewing techniques can be used with PWID. Brief and extended brief interventions are recommended by the National Institute for Health and Clinical Excellence (NICE 2011) for the general population. A feasibility study into an Extended Brief Intervention targeted at PWID has been conducted (Kouimtsidis 2017). Although this study had difficulties recruiting participants from the community intellectual disability and social care services, and did not demonstrate advantage over usual care, it showed that the intervention is acceptable to both clients and carers. To that effect, a large-scale randomised controlled trial recruiting from primary care is needed to establish clinical and cost-effectiveness.

In terms of medications for alcohol use disorders, intellectual disability on its own should not affect decisions to use benzodiazepines in detoxification, nor naltrexone and acamprosate to control drinking. However, it is suggested by the authors that other comorbidities may be present and should be taken into account (e.g. liver function in the use of benzodiazepines). It should be noted that detoxification/ treatment of withdrawal can potentially be more problematic in this patient group because of communication difficulties, lack of assessment tools accessible to this population, physical and psychiatric comorbidities, difficulties in obtaining a reliable alcohol history and possible issues with incapacity to consent to treatment (Miller 2015). Given that PWID are more likely to have carers to prompt adherence to medication, this may allow for successful use of disulfiram. However, given the significant risk in consuming alcohol on disulfiram, even that found in medications and food, the decision to prescribe this should only be made with input from addiction services, a careful risk-benefit analysis, a capacity assessment and close joint working between intellectual disability and addiction services (Miller 2015).

\section{Use of compulsory measures for treatment}

The management of PWID who have alcohol use disorders can lead to ethical dilemmas in balancing a person's right to make decisions about their life, including negative ones, against the duty of care in protecting a group of vulnerable people from potential harm. Another issue is when a person is deemed to lack the capacity to choose to use substances, and whether incapacity legislation could be used to provide them with compulsory treatment for alcohol use disorders. In Scotland, a guardianship with powers to control access to alcohol can be granted for an adult with incapacity. An adult with incapacity is someone aged over 16 years who, by reason of mental disorder or inability to communicate because of physical disability, is incapable of acting, making decisions, communicating decisions, understanding decisions or retaining memory of decisions (HM Government 2000). A mental disorder is defined as a mental illness, personality disorder or learning (i.e. intellectual) disability however caused or manifested, but there is a list of exclusions that includes use of, or dependency on 
MCO answers

$\begin{array}{lllll}1 \mathrm{c} & 2 \mathrm{~b} & 3 \mathrm{~d} & 4 \mathrm{~b} & 5 \mathrm{c}\end{array}$ alcohol or drugs. People with impaired mental functioning caused by past alcohol or drug use can be considered to have a mental disorder, but not those who are temporarily under the influence of alcohol or drugs. Legislation in Scotland therefore excludes substance use or dependency alone from being used as a mental disorder to justify a guardianship order or detention under the Mental Health (Care and Treatment) (Scotland) Act 2003, however, intellectual disability can be used.

In England, incapacity is the inability to make a decision for oneself as a result of being unable to understand the information relevant to the decision, retain that information, use or weigh that information as part of the process of making the decision or communicate his decision (HM Government 2005a) 'because of an impairment of, or a disturbance in the functioning of, the mind or brain' (HM Government 2005b). An impairment can be the temporary effects of alcohol (Department for Constitutional Affairs 2007), although not chronic substance use disorders. The Mental Capacity Act 2005 (MCA) can be used to restrict the access of a PWID to alcohol if it is deemed to be in that person's 'best interests', but the Mental Health Act 2007 can only be used to manage alcohol use disorders if mental illness is also present. Actively restricting someone's access to alcohol in the community would require use of the Deprivation of Liberty Safeguards (an amendment to the MCA, allowing restraint and restrictions that amount to a deprivation of liberty to be used in hospitals and care homes), which would involve an application to the supervising authority if the person lived in a care home, or to the Court of Protection (a body created by the MCA that can make decisions on financial and welfare matters for those lacking capacity) if they were in another setting (Social Care Institute for Excellence 2017).

Although not yet implemented, the Mental Capacity Act (Northern Ireland) 2016 has a similar definition of incapacity and best interests to the MCA 2005, although incapacity is not determined by the presence of a mental disorder and thus would not require a diagnosis of intellectual disability. Actively restricting someone's access to alcohol would likely affect their day-to-day life and therefore be deemed a 'treatment with serious consequences', which would need to be authorised by to the relevant Health and Social Care trust and meet the criteria for prevention of serious harm. Restrictive treatment to control someone's alcohol use would also likely require a second opinion on capacity and best interests, owing to it being a treatment with serious consequences, and also in some cases where the question of whether it is in the person's best interests to have the treatment is 'finely balanced.' (HM Government 2016).
Determining to what extent the unwise decisionmaking in misusing substances can be attributed to the intellectual disability versus the addiction requires careful judgement to ensure that those who truly lack capacity are provided with a legislative framework to keep them safe, and that those who have capacity do not have their right to make unwise decisions disregarded because they have an intellectual disability. Indeed, this is reflected in the concept of best interests under the MCA, where decisions made on behalf of the person who lacks capacity are to be made with consideration of the person's past and present wishes and feelings, and the beliefs and values that would be likely to influence their decision if they had capacity. In Scotland, there is no best interests concept in the legislation, but any decisions made on behalf of an adult with incapacity must benefit the adult, be the least restrictive option and take into account the present and past wishes and feelings of the adult so far as they can be ascertained, and the views of the nearest relative/carer.

Research into how incapacity legislation is currently being used to manage alcohol use disorders in this population would be helpful as there are no published studies on this area, nor any specific guidance available in the UK. The first author is aware, through her own clinical practice in Scotland, of examples where PWID have been placed under welfare guardianship with powers to limit their access to alcohol or drugs. Although this can be beneficial to the patient, it can be extremely difficult to enforce in more capable PWID, and the feasibility of such measures should be considered carefully. Indeed, to enforce such powers effectively usually requires $24 \mathrm{~h}$ supervision, and anecdotally, most cases that the first author has come across are patients under welfare guardianship with high support needs owing to a number of risk factors, of which alcohol use disorders are only a component (e.g. PWID who are violent offenders). Because of their complexity, cases that may require the use of capacity legislation should be assessed and managed jointly between addiction and intellectual disability services.

\section{Conclusions}

Despite concern about the increased risk to PWID from alcohol use disorders with the recent change from institutional to community living, the evidence base is limited with regards to the prevalence of the problem and the strategies used to intervene. It is yet another area in which PWID continue to experience health inequality. It is crucial that health and social care staff are vigilant to ensure alcohol use disorders do not go undetected in this group, and that better joint working between intellectual disability and addiction services occurs to maximise the success of treatment. 


\section{References}

Asscher JJ, van der Put CE, Stams GJ (2012) Differences between juvenile offenders with and without intellectual disability in offense type and risk factors. Research in Developmental Disabilities, 33(6): 1905-13.

Babor TF, Higgins-Biddle JC, Saunders JB, Monteiro MG (2001) AUDIT: The Alcohol Use Disorders Identification Test Guidelines for Use in Primary Care (second edition). World Health Organisation (http://www. who.int/substance_abuse/publications/audit/en/).

Barrett N, Paschos D (2006) Alcohol-related problems in adolescents and adults with intellectual disabilities. Current Opinion in Psychiatry, 19(5): 481-5.

Bhandari A, van Dooren K, Eastgate G, et al (2015) Comparison of social circumstances, substance use and substance-related harm in soon-to-bereleased prisoners with and without intellectual disability. Journal of Intellectual Disability Research, 59(6): 571-9.

Burgard JF, Donohue B, Azrin NH, et al (2000) Prevalence and treatment of substance abuse in the mentally retarded population: an empirical review. Journal of Psychoactive Drugs, 32(3): 293-8.

Caton S, Chadwick D, Chapman M, et al (2012) Healthy lifestyles for adults with intellectual disability: knowledge, barriers, and facilitators. Journal of Intellectual and Developmental Disability, 37(3): 248-59.

Chaplin E, Gilvarry C, Tsakanikos E (2011) Recreational substance use patterns and co-morbid psychopathology in adults with intellectual disability. Research in Developmental Disabilities, 32(6): 2981-6.

Cooper SA (2004) People with intellectual disabilities: their health needs differ and need to be recognised and met. BMJ, 329(7463): 414-5.

Cooper SA, Smiley E, Morrison J, et al (2007) Mental ill-health in adults with intellectual disabilities: prevalence and associated factors. British Journal of Psychiatry, 190(1): 27-35.

Degenhardt L (2000) Interventions for people with alcohol use disorders and an intellectual disability: a review of the literature. Journal of Intellectual and Developmental Disability, 25(2): 135-46.

Department for Constitutional Affairs (2007) Mental Capacity Act 2005 Code of Practice. Department for Constitutional Affairs (https://www. gov.uk/government/uploads/system/uploads/attachment_data/file/497253/ Mental-capacity-act-code-of-practice.pdf).

Ewing J (1984) Detecting alcoholism. The CAGE questionnaire. Journal of the American Medical Association, 252(14): 1905-7.

Forbat L (1999) Developing an alcohol awareness course for clients with a learning disability. British Journal of Learning Disabilities, 27(1): 16-9.

HM Government (2000) Adults with Incapacity (Scotland) Act 2000. TSO (The Stationery Office) (http://www.legislation.gov.uk/asp/2000/4/section/1).

HM Government (2005a) Section 3. Mental Capacity Act 2005. TSO (The Stationery Office) (http://www.legislation.gov.uk/ukpga/2005/9/section/3).

HM Government (2005b) Section 2. Mental Capacity Act 2005. TSO (The Stationery Office) (http://www.legislation.gov.uk/ukpga/2005/9/section/2).

HM Government (2016) Mental Capacity Act (Northern Ireland) 2016. TSO (The Stationery Office) (http://www.legislation.gov.uk/nia/2016/18/contents/enacted).

Huxley A, Copello A, Day E (2005) Substance misuse and the need for integrated services. Learning Disability Practice, 8(6): 14-7.

Kouimtsidis C, Fodor-Wynne L, Scior K, et al (2015) Extended brief intervention to address alcohol misuse in people with mild to moderate intellectual disabilities living in the community (EBI-ID): study protocol for a randomised controlled trial. Trials, 16: 114.

Kouimtsidis C, Bosco A, Scior K, et al (2017) A feasibility randomised controlled trial of extended brief intervention for alcohol misuse in adults with mild to moderate intellectual disabilities living in the community. The EBILD Study. Trials, 18: 216

Kuijken NM, Naaldenberg J, Nijhuis-van der Sanden MW, et al (2016) Healthy living according to adults with intellectual disabilities: towards tailoring health promotion initiatives. Journal of Intellectual Disability Research, 60(3): 228-41.
Lindsay WR, Holland AJ, Carson D, et al (2013) Responsivity to criminogenic need in forensic intellectual disability services. Journal of Intellectual Disability Research, 57(2): 172-81.

Lunsky Y, Elserafi J (2011) Life events and emergency department visits in response to crisis in individuals with intellectual disabilities. Journal of Intellectual Disability Research, 55(7): 714-8.

McCusker C, Clare ICH, Cullen C, et al (1993) Alcohol-related knowledge and attitudes in people with a mild learning disability - the effects of a 'sensible drinking' group. Journal of Community \& Applied Social Psychology, 3(1): 29-40.

McGillicuddy NB, Blane HT (1999) Substance use in individuals with mental retardation. Addictive Behaviors, 24(6): 869-78.

McGillion J, Wanigaratne S, Feinmann C, et al (2000) GP's attitude towards the treatment of drug misusers. British Journal of General Practice, 50(454): 385-6.

McGillivray JA, Moore MR (2001) Substance use by offenders with mild intellectual disability. Journal of Intellectual and Developmental Disability, 26(4): 297-310.

McGillivray JA, Gaskin CJ, Newton DC, et al (2016) Substance use, offending, and participation in alcohol and drug treatment programmes: a comparison of prisoners with and without intellectual disabilities. Journal of Applied Research in Intellectual Disabilities, 29(3): 289-94.

McLaughlin DF, Taggart L, Quinn B, et al (2007) The experiences of professionals who care for people with intellectual disability who have substance-related problems. Journal of Substance Use, 12(2): 133-43.

McLean J, Christie S, Gray L (2017) The Scottish Health Survey 2016 edition, volume 1, main report. Scottish Government (http://www.gov. scot/Publications/2017/10/2970/0)

McMurran M, Lismore K (1993) Using video-tapes in alcohol interventions for people with intellectual disabilities: an exploratory study. British Journal of Learning Disabilities, 21(1): 29-31.

Mendel E, Hipkins J (2002) Motivating learning disabled offenders with alcohol-related problems: a pilot study. British Journal of Learning Disabilities, 30(4): 153-8.

Miller H (2015) Alcohol use disorders. In The Frith Prescribing Guidelines for People with Intellectual Disability (3rd edn) (eds S Bhaumik, SK Gangadharan, D Branford, M Barrett): 203-14. John Wiley \& Sons.

National Institute for Health and Clinical Excellence (NICE) (2011) AlcoholUse Disorders: Diagnosis, Assessment and Management of Harmful Drinking and Alcohol Dependence. NICE (https://www.nice.org.uk/guidance/cg115).

NHS Health Scotland (2004) Health Needs Assessment Report. People with Learning Disabilities in Scotland. NHS Health Scotland (http:// www.gla.ac.uk/media/media_63872_en.pdf).

Pezzoni V, Kouimtsidis C (2015) Screening for alcohol misuse within people attending a psychiatric intellectual disability community service. Journal of Intellectual Disability Research, 59(4): 353-9.

Robertson J, Emerson E, Gregory N, et al (2000) Lifestyle related risk factors for poor health in residential settings for people with intellectual disabilities. Research in Developmental Disabilities, 21(6): 469-86.

Scott D, McGilloway S, Donnelly M (2006) The mental health needs of people with a learning disability detained in police custody. Medicine, Science and the Law, 46(2): 111-4.

Scottish Government (2013) The Keys to Life: Improving Quality of Life for People with Learning Disabilities. The Scottish Government (https://key stolife.info/wp-content/uploads/2014/05/the-keys-to-life-full-version.pdf).

Slayter E (2010) Medicaid-covered alcohol and drug treatment use among people with intellectual disabilities: evidence of disparities. Intellectual and Developmental Disability, 48(5): 361-74

Social Care Institute for Excellence (2017) Deprivation of Liberty Safeguards (DoLS) at a Glance. Social Care Institute for Excellence (https://www.scie.org.uk/mca/dols/at-a-glance).

Taggart L, McLaughlin D, Quinn B, et al (2006) An exploration of substance misuse in people with intellectual disabilities. Journal of Intellectual Disability Research, 50(Pt 8): 588-97. 
Taggart L, McLaughlin D, Quinn B, et al (2007) Listening to people with intellectual disabilities who misuse alcohol and drugs. Health and Social Care in the Community, 15(4): 360-8.

To WT, Neirynck S, Vanderplasschen W, et al (2014) Substance use and misuse in persons with intellectual disabilities (ID): results of a survey in ID and addiction services in Flanders. Research in Developmental Disabilities, 35(1): 1-9.

VanDerNagel J, Kiewik M, Buitelaar J, et al (2011) Staff perspectives of substance use and misuse among adults with Intellectual Disabilities Enrolled in Dutch Disability Services. Journal of Policy and Practice in Intellectual Disabilities, 8(3): 143-9.
Van Straaten B, Schrijvers CT, Van der Laan J, et al (2014) Intellectual disability among Dutch homeless people: prevalence and related psychosocial problems. PLoS ONE, 9(1): e86112.

Westermeyer J., Kemp K., Nugent S (1996) Substance disorder among persons with mild mental retardation: a comparative study. The American Journal on Addictions, 5(1): 23-31.

World Health Organization (2010) ICD-10 Version: 2010. WHO (http:// apps.who.int/classifications/icd10/browse/2010/en).

World Health Organization (2017) ICD-11 Beta Draft. WHO (https://icd. who.int/dev11//-m/en)

\section{MCOs}

Select the single best option for each question stem

1 The prevalence of alcohol use disorders in PWID in the UK:

a is higher than in the general population

$\mathrm{b}$ is thought to be particularly high in females

c is unclear but is probably lower than in the general population

$d$ has been reliably measured in studies to date

e has decreased with the move from institutional to community care.

\section{PWID who misuse alcohol:}

a are less vulnerable to the physical effects of alcohol use

b are not a homogeneous group and thus a successful intervention requires an individually tailored approach

c are less likely to experience psychological consequences of drinking

d should adhere to stricter drinking limits than people without intellectual disability as there is strong evidence to recommend what these limits are

e find addiction services easily accessible.
3 Concerning the services available for PWID with alcohol use disorders:

a intellectual disability services are very experienced and confident in supporting this group

b addiction services are very experienced and confident in supporting this group

c joint working between services is well established

d PWID report difficulties in accessing addiction support from both addiction and intellectual disability services

e research has indicated that third-sector organisations are well-equipped to support this group.

4 Regarding management of alcohol use disorders in PWID:

a there is a lot of evidence regarding the efficacy of interventions targeted specifically at this population

b there are a number of ways in which interventions can be adapted to improve accessibility for this population

c highly cognitive-based therapies are particularly suited to this patient group

d motivational interviewing techniques are impossible with this patient group

e medical management is not helpful in this patient group.
5 Regarding legislative frameworks for managing PWID who misuse alcohol:

a alcohol use disorder alone is a sufficient diagnosis to support a finding of incapacity regarding personal welfare

b use of compulsory measures to manage substance use disorders are readily enforceable even in more capable PWID

c decisions regarding capacity to misuse substances should be made with care in this population to discern whether the addiction or intellectual disability is driving the decisionmaking

d PWID should be protected from all risk-taking behaviour

e incapacity legislation in the UK does not encompass powers to control access to substances. 